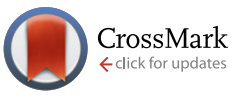

Cite this: Med. Chem. Commun., 2016, 7, 1176

Received 23rd December 2015, Accepted 21st April 2016

DOI: $10.1039 / c 5 m d 00596 e$

www.rsc.org/medchemcomm

\title{
The antidepressant drug paroxetine as a new lead candidate in schistosome drug discovery $\dagger$
}

\author{
Bruno Junior Neves, $t^{\mathrm{t}}$ Rafael Ferreira Dantas, $\hat{t}^{\mathrm{b}}$ Mario Roberto Senger, ${ }^{\mathrm{b}}$

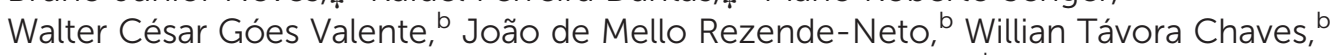 \\ Lee Kamentsky, ${ }^{\mathrm{C}}$ Anne Carpenter, ${ }^{C}$ Floriano Paes Silva-Junior ${ }^{\star b}$ \\ and Carolina Horta Andrade*a
}

\begin{abstract}
Recently, our in silico repositioning-chemogenomics approach predicted paroxetine (PAR), an antidepressant drug, as a inhibitor of Schistosoma mansoni serotonin transporters (SmSERTs), and consequently, a new anti-schistosomal candidate. With the aim of determining the anti-schistosomal activity of this drug, we initially used a spectrophotometric assay to determine activity against schistosomula worms. During this investigation, we verified that PAR showed a pronounced effect on schistosomula viability $\left(\mathrm{IC}_{50}=2.5 \mu \mathrm{M}\right)$ after $72 \mathrm{~h}$ of incubation. Then, we performed ex vivo studies with adult S. mansoni worms using a new automated image-based assay to accurately measure worm motility. As expected from the PAR's predicted mechanism of action, both male and female worms treated with low concentrations of PAR exhibited enhanced motility followed by reduction in motility as incubation time increased. PAR EC 50 values for motility reduction in male and female worms were $5.1 \mu \mathrm{M}$ and $9.9 \mu \mathrm{M}$ after $24 \mathrm{~h}$ of exposure, respectively, and this effect was maintained until the end of the experiment (72 h). Lastly, homology modeling and docking studies with SmSERT-A and human SERT (hSERT) revealed insights into the chemical basis of PAR antischistosomal activity. These results provide crucial guidance for further studies to optimize PAR in terms of potency and selectivity.
\end{abstract}

\section{Introduction}

Schistosomiasis is a neglected tropical disease caused by flatworms of the genus Schistosoma, with three species (S. mansoni, $S$. haematobium, and $S$. japonicum) accounting for the majority of human infections. These worms cause a chronic and often debilitating infection that impairs development and productivity, and this exposure is strongly linked to extreme poverty. ${ }^{1-4}$ Recent estimates suggest that around 262 million people are infected, mainly in sub-Saharan Africa, the Middle East, the Caribbean, and South America, resulting in up to 200000 deaths annually, while more than 42 million infected individuals experience high morbidity. ${ }^{5}$

In the absence of an effective vaccine, the control of schistosomiasis relies on a single drug, praziquantel (PZQ), which

\footnotetext{
${ }^{a}$ LabMol - Laboratory for Molecular Modeling and Design, Faculdade de Farmácia, Universidade Federal de Goiás, Goiânia - GO, Brazil. E-mail: carolina@ufg.br ${ }^{b}$ LaBECFar - Laboratório de Bioquimica Experimental e Computacional de Fármacos, Instituto Oswaldo Cruz, Fundação Oswaldo Cruz, Rio de Janeiro - RJ, Brazil. E-mail: floriano@ioc.fiocruz.br

${ }^{c}$ Imaging Platform, Broad Institute of Massachusetts Institute of Technology and

Harvard, Cambridge, Massachusetts, USA

$\dagger$ The authors declare no competing interests.

$\$$ These authors contributed equally.
}

has been used in mass drug administration programs for almost four decades. ${ }^{6}$ However, PZQ has low efficacy against juvenile worms, found during the early stages of infection (14-28 day post infection). ${ }^{6-9}$ Moreover, reports of PZQ resistant or poorly sensitive isolates in African countries have been published ${ }^{10-12}$ and a resistant strain was also induced in the laboratory. ${ }^{13-15}$ Consequently, there are concerns that the widespread use of PZQ could potentially lead to development of resistant parasites in the near future. Hence, there is an urgent need for discovering new anti-schistosomal drugs with new mechanisms of action.

Recently, using an in silico repositioning-chemogenomics approach, ${ }^{16}$ our group predicted paroxetine (PAR), an antidepressant drug, as a $S$. mansoni serotonin transporter (SmSERT) inhibitor. Studies on the physiological functions of plasma membrane SERT were first focused on the mammalian central nervous system, where it mediates re-uptake of the amine across the presynaptic membrane. ${ }^{17}$ This is part of a mechanism that inactivates serotonin signalling by quickly removing it from the synaptic cleft. ${ }^{18}$

Studies dating back to the 1970s and 1980s have shown that among parasitic flatworms, in particularly S. mansoni, serotonin is an important modulator of neuromuscular function and increases metabolic activity by stimulating glucose 
uptake, glycogen breakdown, and lactate excretion. ${ }^{19-22}$ The effects on motility are seen in intact, unpermeabilized worms, showing that serotonin either acts through surface receptors, or is taken internally via a specific transporter and acts directly on the worm musculature. ${ }^{23}$ More recently, the existence of two SmSERT isoforms was confirmed by molecular methods. These isoforms are structurally similar to other SERTs except for the presence of an additional 78 amino acids at the $\mathrm{N}$-terminal end, which is presumably due to differential splicing of the transcript. In addition, both isoforms were cloned from $S$. mansoni, expressed heterologously in mammalian cells and shown to have selective, high-affinity serotonin transporter activity with a $\mathrm{Km}$ value comparable to that of mammalian SERTs. ${ }^{23,24}$ This suggests that $S$. mansoni is dependent on SmSERTs to regulate serotonin concentrations in the synaptic cleft and consequently its neuromuscular function. ${ }^{23-25}$

In this study, we validated the anti-schistosomal activity of PAR on schistosomula and adult $S$. mansoni worms using a spectrophotometric assay and a new automated image-based assay to accurately measure parasite viability and motility, respectively. Further, to guide the design and optimization of new anti-schistosomal drugs, we explored the interactions of PAR with the binding site of SmSERTs and its respective host counterpart ( $h$ SERT) using molecular modeling strategies.

\section{Results and discussion}

\section{Biological activity}

The lack of activity against immature worms is a therapeutic limitation of PZQ which hampers the treatment in endemic areas since it does not prevent re-infection. ${ }^{26}$ Therefore, we used a spectrophotometric, i.e., resazurin-based assay, to determine the viability of schistosomula after the exposure to PAR for $72 \mathrm{~h}$ incubation period. This approach is commonly used as an initial screening step in antischistosomal lead discovery campaigns, ${ }^{27-32}$ since schistosomula are relatively easier to obtain in larger amount when compared with adult worms. This analysis showed that PAR decreased schistosomula viability after $72 \mathrm{~h}$ of incubation $\left(\mathrm{IC}_{50}=2.5 \mu \mathrm{M}\right)$ while PZQ had only negligible activity against this parasite stage at concentrations as high as $32 \mu \mathrm{M} .^{33}$ Remarkably, PAR effect on schistosomula viability corroborates with results of a screening campaign with 2160 compounds that identified several $h$ SERT inhibitors as potent hits for $S$. mansoni schistosomula. ${ }^{32}$

After the initial screening, separated individual adult male and female $S$. mansoni worms were incubated with PAR for various time periods up to $72 \mathrm{~h}$. Consistent and objective quantification of anti-schistosomal activity relied on an automated image-based motility assay; especially important given the limited numbers of adult worms that can be feasibly analysed and the tedious procedure for culturing and isolating them. As expected from the hypothesized mechanism of action for PAR in schistosomes, we observed for low drug concentrations an increase in worm motility of up to 9-fold in females and 2-fold in males immediately after addition of the drug in the culture medium, i.e., time $0 \mathrm{~h}$ (see Fig. 1).

This transient excitation in worm motility was only detected for the lower part of the tested drug concentration range $(\leq 10 \mu \mathrm{M}$ in females and $\leq 20 \mu \mathrm{M}$ in males). This characteristic corroborates the effect produced by two classical serotonin transport inhibitors, fluoxetine and clomipramine, on schistosomula which were previously shown to induce a strongly hyperactive phenotype, corresponding to a 3-fold increase in larval motility, roughly the same effect as treatment with an excess of exogenous serotonin. ${ }^{34}$ On the other hand, higher concentrations may be extremely toxic to the parasite causing immediate viability loss and a consequent reduction in worm motility. After $24 \mathrm{~h}$ incubation this hypermotility effect is reversed, with schistosomes showing decreased motility, which is expected to also be associated with diminished viability. This delayed effect would be consistent with the timing of serotonin receptor internalisation, a protective mechanism to avoid excitotoxicity. ${ }^{35}$

Using worm motility measurements obtained from our automated image-based assay, dose-response curves were also generated for each incubation time as exemplified in Fig. 2 for the $24 \mathrm{~h}$ time point.

The $\mathrm{EC}_{50}$ values determined for PAR (Table 1) indicate that after $24 \mathrm{~h}$ incubation the effect is fully developed with values varying from 2.7 to $5.1 \mu \mathrm{M}$ for male worms and 9.9 to 11.9 $\mu \mathrm{M}$ for female worms. The effect of PAR on adult males after $72 \mathrm{~h}$ of exposure is in the same range of the value determined for schistosomula. Our results also indicate that male worms are slightly more sensitive to PAR action since they showed on average $\mathrm{EC}_{50}$ values $2-3$ times lower than those determined in females. In part, this could be due to a gender-specific expression pattern of SmSERT. ${ }^{34}$

A

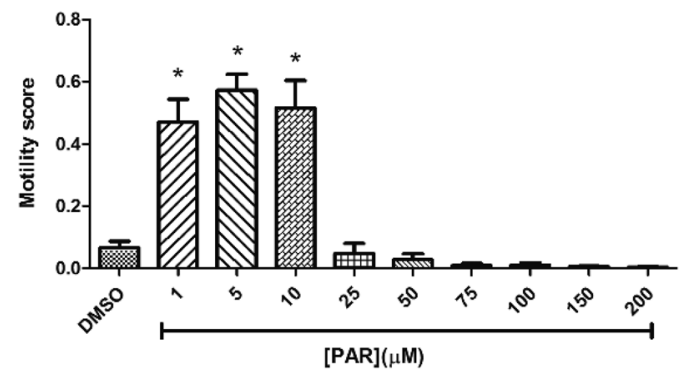

B

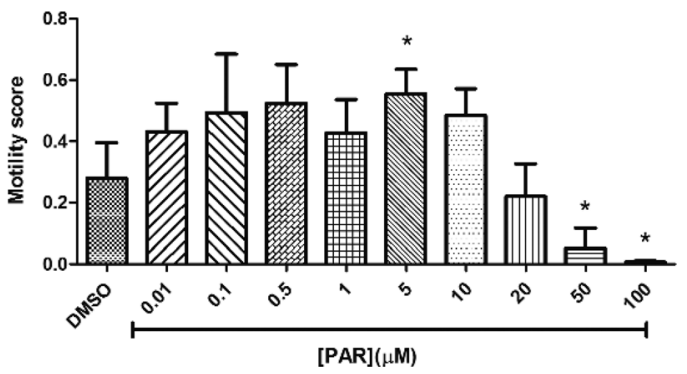

Fig. 1 PAR effect on motility of adult female (A) and male (B) $S$. mansoni worms immediately after addition to culture medium $(0 \mathrm{~h})$. Data expressed as mean \pm standard deviation. * Statistically different from DMSO control ( $p$-value $<0.05)$. 
A

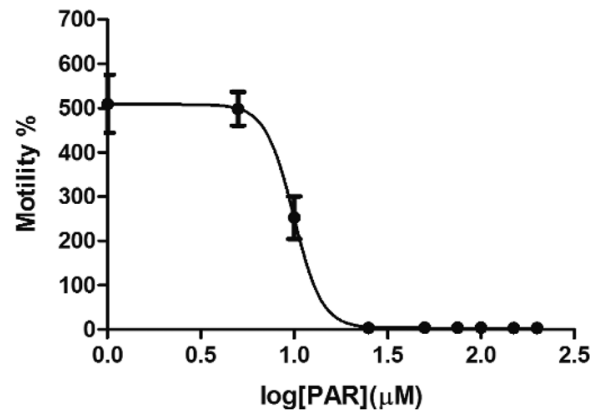

B

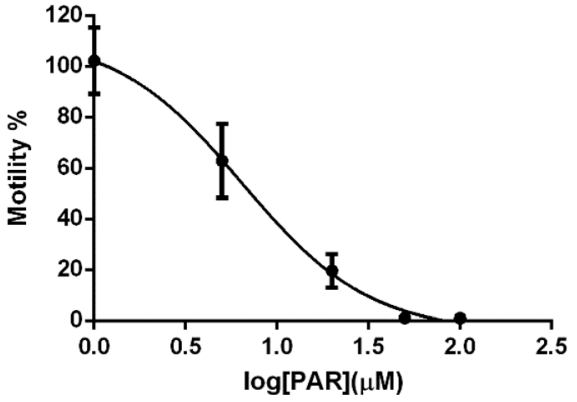

Fig. 2 PAR dose-response curves in female (A) and male (B) S. mansoni adult worms after $24 \mathrm{~h}$ of incubation. Data expressed as mean \pm standard error for the mean. Percent values were calculated using the motility mean of the control group (DMSO $0.2 \%$ ). PAR concentrations that increased worms motility are not shown in male dose-response curve.

Table 1 Time- and gender dependent $\mathrm{EC}_{50}$ values $(\mu \mathrm{M})$ for PAR and PZQ

\begin{tabular}{lrrrll}
\hline & \multicolumn{2}{c}{ PAR } & & \multicolumn{2}{c}{ PZQ } \\
\cline { 2 - 3 } \cline { 6 - 6 } Time/gender & Male & Female & & Male & Female \\
\hline $0 \mathrm{~h}$ & 23.5 & 17.4 & & 0.11 & 0.13 \\
$24 \mathrm{~h}$ & 5.1 & 9.9 & & 0.12 & 0.30 \\
$48 \mathrm{~h}$ & 3.8 & 11.5 & & 0.13 & No fit \\
$72 \mathrm{~h}$ & 2.7 & 11.9 & & No fit & 0.66
\end{tabular}

On the other hand, $\mathrm{EC}_{50}$ values indicate that PAR had a less pronounced effect on $S$. mansoni motility than PZQ at all incubation times $\left(\mathrm{EC}_{50}\right.$ values $\leq 0.66 \mu \mathrm{M}$, see Table 1$)$. These results are in general accordance with the $\mathbf{E C}_{50}$ value described for PZQ $(0.2 \mu \mathrm{M})$ after $72 \mathrm{~h}$ of exposure using a visual analysis. $^{30}$ However, the alternate mechanism of action of PAR (and status as clinically-approved for human use) makes it an interesting molecular scaffold to develop more efficient compounds. Additionally, in contrast to PZQ, for which the molecular target remains unknown, new analogs of PAR may be rationally designed using $3 \mathrm{D}$ structural information of SERTs during the chemical optimization process (see docking results below).

\section{Homology modelling of SmSERTs and hSERT}

We developed homology models of SmSERTs and hSERT to shed some light into PAR's binding mode and to explore structural differences between the two isoforms of SmSERT and the human enzyme. The two SmSERT isoforms differed in only three amino acids located in the protein's predicted intracellular N-terminal. SmSERT-A has Leu99, Glu100 and
Val118 while the corresponding amino acid residues in SmSERT-B are Ser99, His100 and Iso118. $^{24}$ Initially, we selected suitable template protein structures in Protein Data Bank (PDB), ${ }^{36}$ observing the following criteria: the template should have a high coverage $(>70 \%$ of target aligned to template), sequence identity $>30 \%$, and X-ray crystallography resolution $\leq 3.0 \AA$. The SmSERT-A and SmSERT-B were built using Drosophila melanogaster dopamine transporter $(\text { DmDAT })^{37,38}$ available under PDB code $4 \mathrm{M} 48$ (sequence identity $=54.5 \%$, and coverage $=73.3 \%$ ). The $h$ SERT structure was also modelled using DmDAT available under PDB code $4 \mathrm{M} 48$, (sequence identity $=53.3 \%$ and coverage $=85.0 \%$ ).

In order to identify structural distances between the modeled structures and their templates, the SmSERT-A, SmSERT-B, and hSERT models were superimposed onto their corresponding templates and root-mean-square deviations (RMSD) between backbone $\mathrm{C} \alpha$-atoms were calculated. The SmSERT models showed a RMSD value of $0.19 \AA$ (Fig. 3A), while the hSERT model showed an RMSD of $0.06 \AA$ (Fig. 3B). Most of the differences between the models and their templates were observed in the extracellular flexible loop. Remarkably, this extracellular loop of SmSERTs is exceptionally long compared to the $h$ SERT and therefore biologically relevant, but this region is far from the binding site of SERTs ${ }^{23}$ and it is unlikely that these differences will affect the docking studies performed in the binding site. In addition, we did not observe structural and conformational differences between binding sites of SmSERTs. Therefore, we decided to keep only the SmSERT-A structure for the next modelling experiments. In order to evaluate the stereochemical quality of dihedral angles phi against psi of amino acid residues in the modeled protein structures and to identify sterically allowed regions for these angles, we used the PROCHECK analysis. Analysis of SmSERT-A protein revealed that $93.8 \%$ residues are within the most favoured regions (red), 5.4\% in additional allowed regions (yellow), $0.6 \%$ residues in generously allowed regions (beige), and only $0.2 \%$ residues in the disallowed regions (white) of the Ramachandran plot (Fig. 3C), showing the good quality of the generated homology model. Similarly, for $h$ SERT structure the observed values were $93.1 \%, 5.9 \%$, $0.8 \%$, and $0.2 \%$, respectively (Fig. 3D). Residues located in the disallowed regions are far from the binding site of both models, indicating that these residues may not affect the ligand-protein binding simulations. The quality of the homology models was further evaluated by the ERRAT scores of 80.8 (SmSERT-A) and 87.2 ( $h$ SERT), which indicated an acceptable protein environment for the non-bonded interactions between different atom types.

\section{Molecular docking of PAR}

To explore the possible intermolecular interactions of PAR in the binding sites of SmSERT-A and hSERT, we performed molecular docking. Docking results showed that the predicted binding modes of PAR in both targets are similar with the experimental binding mode of the co-crystalized ligand of X-ray structure used as template in homology modeling. ${ }^{32,33}$ In particular, the 
A

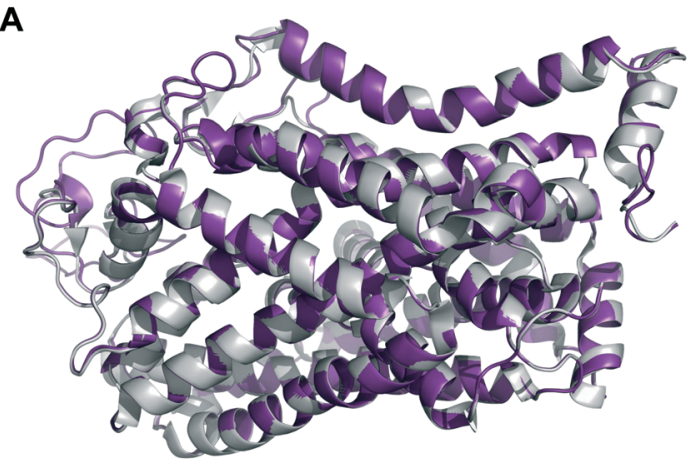

C

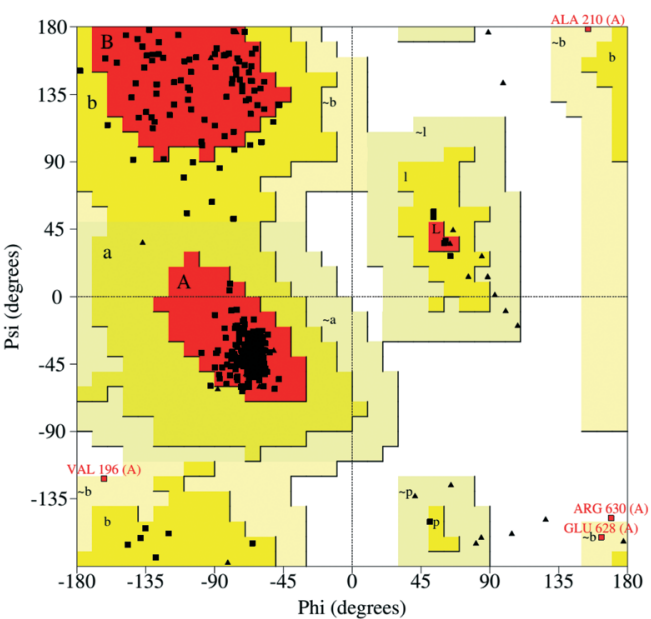

B

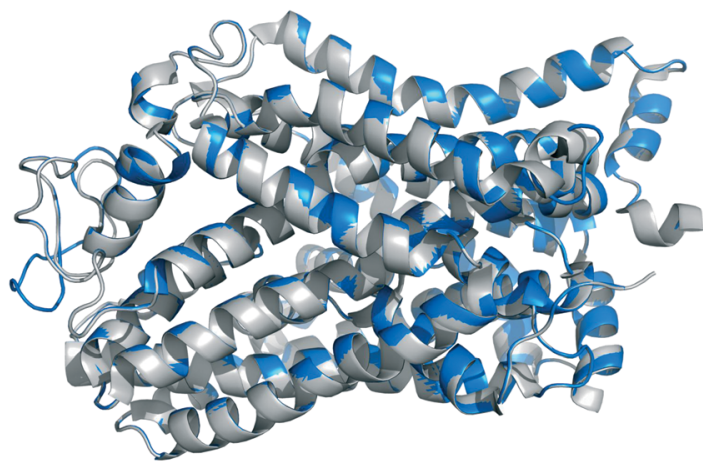

D

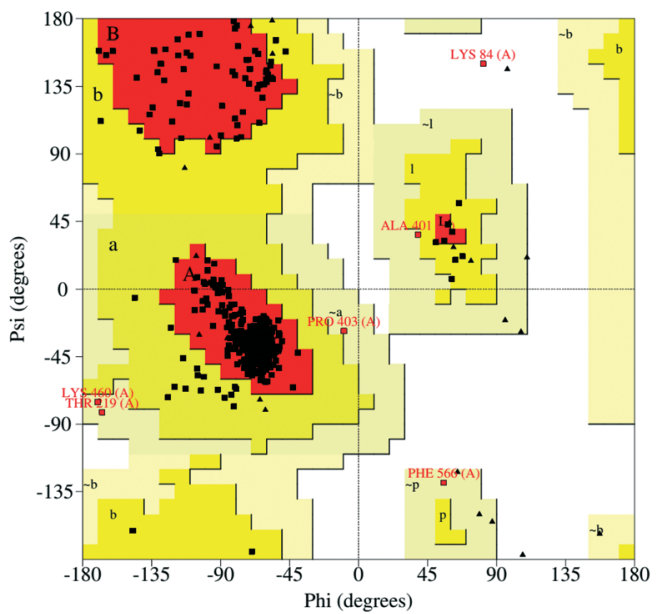

Fig. 3 Superimposition of modeled SmSERT-A (A, purple) and $h$ SERT (B, blue) with their respective templates (gray). Ramachandran plots for the SmSERT-A model (C) and hSERT model (D) obtained by PROCHECK, showing the dihedral angles Psi and Phi of amino acid residues. Red represents most favoured regions; yellow represents additional allowed regions; beige represents generously allowed regions; and white areas are disallowed regions.

aromatic rings of PAR interact with the hydrophobic pocket of SmSERT-A formed by Tyr162, Phe346, Phe352, Ala506, and Thr158, whereas the protonated nitrogen of piperidine is able to form two hydrogen bonds (represented as green dashed lines) with the carbonyl groups of Phe81 and Asp84 (Fig. 4A). The docking of PAR in the binding site of $h$ SERT showed simi- lar interactions (Fig. 4B). Consequently, the predicted binding affinities of PAR by SmSERT-A and $h$ SERT were very similar, -11.3 and -12.6 , respectively, showing that this ligand could have similar affinity with these two SERT proteins. Those observations corroborate the inhibition potencies of PAR measured by Fontana and colleagues ( IC $_{50}$ for SmSERTs around
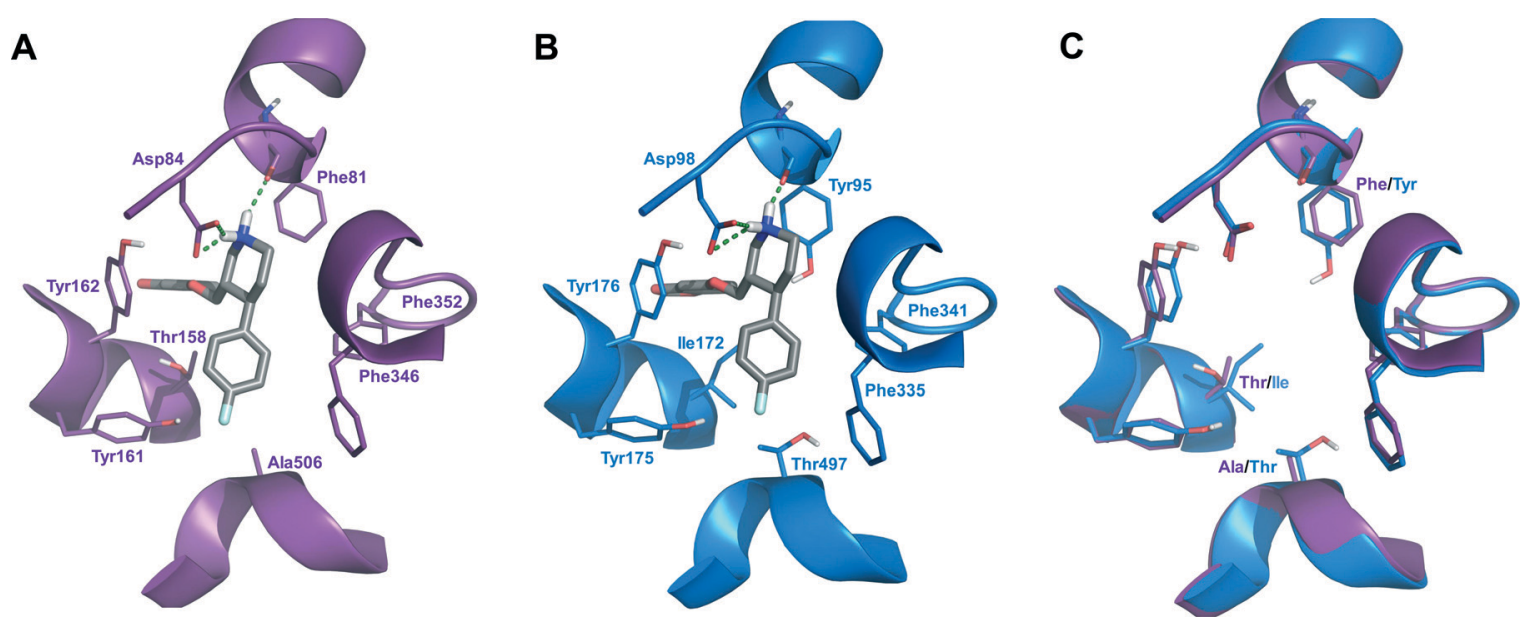

Fig. 4 Intermolecular interactions of SmSERT-A (A) and $h$ SERT (B) with PAR, and structural differences between binding sites (C). 
$0.09 \mu \mathrm{M}$ and $\mathrm{IC}_{50}$ for $h \mathrm{SERT}=0.02 \mu \mathrm{M}$ ) in a heterologous expression system. ${ }^{20}$ It is worth noting that the protein flexibility should be taken into account to further explore the differences in the binding process of PAR with SmSERT-A and hSERT in order to design a more selective SmSERT inhibitor.

In addition, our analysis of the modeled proteins revealed differences in the hydrophobicity of the two binding sites. Several amino acid residues of the binding site (Thr158, Phe81 and Ala506) of SmSERTs were substituted in hSERT by Ile172, Tyr95 and Thr497, respectively (Fig. 4C). It therefore appears that the binding site of SmSERTs can accommodate bulkier ligands. These key differences between SmSERTs and $h$ SERT may be useful to design more potent and selective anti-schistosomal drug candidates.

\section{Experimental}

\section{Reagents}

PAR was purchased from Gemini (Anápolis-GO, Brazil) while PZQ was acquired from Sigma-Aldrich (St. Louis-MO, USA). Both drugs were diluted in $100 \%$ DMSO prior the assays. DMEM medium were purchased from Vitrocell (Campinas-SP, Brazil). All other reagents were acquired from Sigma-Aldrich (St. Louis-MO, USA).

\section{Ethics statement}

Animal maintenance and experiments were carried out in accordance with the Institutional Ethics Committee for Laboratory Animal Use at the Oswaldo Cruz Foundation (CEUA/FIOCRUZ, Brazil; license number LW-78/12).

\section{In vitro schistosomula resazurin-based viability assay}

Cercariae ( $S$. mansoni BH strain) were shed in clean tap water from infected Biomphalaria glabrata snails exposed to direct illumination for $1 \mathrm{~h}$. Cercariae were then vortexed at maximum speed for $5 \mathrm{~min}$ and transformed into schistosomula by an mechanical method adapted from literature. ${ }^{33,39}$ Then, we performed the schistosomula resazurin-based viability assay as described previously, with minimal modifications. ${ }^{33}$ Schistosomula were maintained in a complete M169 medium (120 per well) and incubated in black 384 well plates at $37{ }^{\circ} \mathrm{C}$ and $5 \%$ $\mathrm{CO}_{2}$. The effect of PAR on schistosomula viability was assessed $72 \mathrm{~h}$ after drug exposure at concentrations of $0.05-200 \mu \mathrm{M}$. Lastly, $8 \mu \mathrm{L}$ of resazurin (final concentration: $15 \mu \mathrm{g} \mathrm{mL}{ }^{-1}$ ) was added to each well $24 \mathrm{~h}$ before fluorescence measurement. The fluorescence intensity was measured using a FlexStation 3 Multi-Mode Microplate Reader (Molecular Devices) using an excitation wavelength of $530 \mathrm{~nm}$ and an emission wavelength of $580 \mathrm{~nm}$.

\section{Mice infection and ex vivo assays on adult $S$. mansoni}

Swiss mice (three- to six-days old) were infected percutaneously with $150 \pm 10$ cercariae ( $S$. mansoni $\mathrm{BH}$ strain). The animals were placed, for a period of $30 \mathrm{~min}$, into cylindrical vials under incandescent light with a thin water layer containing the cercariae. After $S$. mansoni maturity (42-49 days after infection), mice were euthanized, and worms were perfused with $0.85 \%$ sodium chloride and $0.75 \%$ sodium citrate solution. Male and female worms were maintained separately into 96 well plates with complete DMEM medium (i.e. DMEM plus $10 \%$ fetal calf serum, $2 \mathrm{mM}$ L-glutamine, $100 \mu \mathrm{M} \mathrm{ml}^{-1}$ penicillin, $100 \mu \mathrm{g} \mathrm{ml}^{-1}$ streptomycin) throughout the entire experiment in an incubator at $37{ }^{\circ} \mathrm{C}$ with $5 \% \mathrm{CO}_{2}$. The effect of PAR (concentrations ranging between 0.01-200 $\mu \mathrm{M}$ ) and positive control (i.e., PZQ incubated with $0.05-1 \mu \mathrm{M}$ ) on adult worms was assessed either immediately or 24,48 or $72 \mathrm{~h}$ after compound addition. Negative control wells contain DMEM with $0.2 \%$ of DMSO. The ex vivo drug sensitivity assay was based on motility measurements obtained with a newly developed anti-helminthic high-content screening platform briefly described below.

\section{Image acquisition and quantitative image analysis}

Our method is based on sequential pairwise comparison of 100 time-lapse images captured every 250-300 ms using an automated bright-field microscope with a $2 \times$ objective lens (ImageXpress Micro XLS, Molecular Devices, CA). Subsequent quantitative image analysis used a custom-developed pipeline for detecting changes in parasite motility using the opensource CellProfiler software version 2.1.2. ${ }^{40}$ The pipeline along with its validation will be thoroughly described in a subsequent publication and the pipeline itself is freely available at www.cellprofiler.org/published_pipelines.shtml.

Briefly, at each cycle of the pipeline, an image captured at a given instant $\left(t_{n}\right)$ is compared with the image captured at the preceding instant $\left(t_{n-1}\right)$ and so on until all images are processed. Two different motility measurements are calculated. First, a precursor metric, "AdjustedRandIndex" is calculated by comparing worm objects identified from images captured at times $t_{n}$ and $t_{n-1}$ with CellProfiler's Overlap module. This measure ranges from 0 to 1 , with 1 meaning two objects are perfectly aligned (no movement). Thereafter, we calculate an "Overlap" mobility score, which is directly proportional to the amount of movement, by subtracting 1 - "AdjustedRandIndex". Another motility measure, "DiffWorms", is the mean pixel intensity of the image calculated from the absolute difference of the parasite images in $t_{n-1}$ and $t_{n}$. A higher DiffWorms score indicates higher parasite mobility. Both measures are iteratively taken for the 99 image pairs, and scores per well are calculated by averaging over all measurements.

\section{Statistical analysis}

All statistical analysis and graphs were performed using GraphPad Prism version 5.00 for Windows (GraphPad Software, La Jolla California USA, www.graphpad.com).

\section{Homology modeling}

The amino acid sequences of SmSERT-A (ID: DQ220811), SmSERT-B (ID: DQ159205) and hSERT (ID: P31645) were retrieved from GenBank database ${ }^{41}$ and used as targets for 
homology modelling using the SWISS-MODEL server. ${ }^{42}$ The latter performed the target-template sequence alignment after searching the putative X-ray template proteins in PDB for generating the $3 \mathrm{D}$ models for both target sequences. The built homology models are prone to contain internal constrains like unfavorable bond lengths, bond angles, torsion angles and contacts. Therefore, built models were exported to SAVES server (http://services.mbi.ucla.edu/SAVES/) and their overall stereochemical and structural quality was checked according to PROCHECK ${ }^{43}$ and ERRAT scores. ${ }^{44}$ PROCEHCK was applied to check the stereochemical quality of the model, including backbone torsional angles through the Ramachandran plot, while ERRAT, by means of its so-called overall quality factor, was used to check non-bonded atomic interactions, with higher scores indicating higher quality.

\section{Docking studies}

The generated homology models were imported into Maestro v. 10.0 (Schrödinger, LLC, New York, NY, 2014) and prepared using the Protein Preparation Wizard workflow as follows: hydrogen atoms were added according to Epik v. $2.7^{45}(\mathrm{pH} 7.4 \pm$ 1.0) and minimized using the OPLS-2005 force field. ${ }^{46}$ Then, structure of PAR was also imported to Maestro and its most favorable ionization state was generated at $\mathrm{pH} 7.4 \pm 1.0 \mathrm{using}$ Epik. Subsequently, 300 conformations were generated using OMEGA v. 2.5.1, ${ }^{47}$ while AM1-BCC charges $^{48}$ were added using QUACPAC v. 1.6.3. ${ }^{49}$

Previous to docking studies, a grid was defined to include the full ligand-binding site of SmSERT, with dimensions of 18.6 $\AA$ × $16.0 \AA \times 17.6 \AA(x, y$, and $z)$ and volume of $5276 \AA^{3}$ around Phe81, Asp84, Phe346, and Phe352 amino acid residues of binding site. The grid of $h$ SERT was generated with dimensions of $17.0 \AA \times 16.3 \AA \times 17.0 \AA$ and volume of $4720 \AA^{3}$ around Asp98, Tyr95, Phe335, and Phe341. Molecular docking of PAR on the built homology models was investigated using FRED software, available in OEDocking suite v. 3.0.1 ${ }^{50,51}$ using ChemGauss4 scoring function. FRED is a docking program that performs an exhaustive search, by systematically searching rotations and translations of each conformer of the ligand within the active site, filtering the possible poses for shape complementarity ${ }^{52}$ and pharmacophoric features before selecting and optimizing poses using the Chemgauss 4 scoring function. ${ }^{50,53}$

\section{Conclusions}

We identified potent in vitro and ex vivo anti-schistosomal activity of PAR against schistosomula and adult life stages of S. mansoni, respectively. This drug offers a new biochemical pathway to kill schistosomes by disrupting serotonin signalling and its downstream events. ${ }^{25,34,54}$ In addition, its mechanism of action may complement those currently postulated for PZQ (i.e., increased $\mathrm{Ca}^{2+}$ influx, inhibition of nucleoside uptake $)^{55}$ and thus could help to overcome resistance. Although SmSERT has not been validated as the definitive target of PAR, it is likely the main mechanism of action on schistosomes. In absence of a structure obtained from X-ray crystallography or NMR experiments, we developed 3D homology models to shed some light on the structural differences between the binding sites of SmSERTs and $h$ SERT, and performed molecular docking studies with SmSERT-A and $h$ SERT to explore the possible interactions of PAR in the binding site of both SERTs. We observed similar predicted binding modes of PAR in both enzymes. In conclusion, we would recommend further medicinal chemistry efforts to optimize PAR scaffold in terms of potency, selectivity, and permeability to the tegument of the parasite.

\section{Acknowledgements}

The authors would like to thank Brazilian funding agencies, CNPq, CAPES, FAPEG, FAPERJ and FIOCRUZ for financial support and fellowships, as well as the US National Institutes of Health (grant NIH GM095672 to AEC). We are grateful to OpenEye Scientific Software Inc. for providing academic license of their software. The authors also thank the Malacology Laboratory (Dr. Silvana C. Thiengo) from IOC/FIOCRUZ for providing $S$. mansoni cercarie and the Bioassays and Drug Screening Platform (FIOCRUZ RPT11-I subunit) for technological support. Special thanks to Molecular Devices for providing acess to HCS equipment.

\section{Notes and references}

1 D. G. Colley, A. L. Bustinduy, W. E. Secor and C. H. King, Lancet, 2014, 383, 2253-2264.

2 A. G. P. Ross, P. B. Bartley, A. C. Sleigh, G. R. Olds, Y. Li, G. M. Williams and D. P. McManus, N. Engl. J. Med., 2002, 346, 1212-1220.

3 C. H. King, N. Engl. J. Med., 2009, 360, 106-109.

4 B. Gryseels, K. Polman, J. Clerinx and L. Kestens, Lancet, 2006, 368, 1106-1118.

5 World Health Organization. Schistosomiasis. Available from: http://www.who.int/mediacentre/factsheets/fs115/en, 2015.

6 R. Gönnert and P. Andrews, Z. Parasitenkd., 1977, 52, 129-150.

7 A. A. Sabah, C. Fletcher, G. Webbe and M. J. Doenhoff, Exp. Parasitol., 1986, 61, 294-303.

8 L. Pica-Mattoccia and D. Cioli, Int. J. Parasitol., 2004, 34, 527-533.

9 A. D. Aragon, R. A. Imani, V. R. Blackburn, P. M. Cupit, S. D. Melman, T. Goronga, T. Webb, E. S. Loker and C. Cunningham, Mol. Biochem. Parasitol., 2009, 164, 57-65.

10 M. Ismail, A. Metwally, A. Farghaly, J. Bruce, L. F. Tao and J. L. Bennett, Am. J. Trop. Med. Hyg., 1996, 55, 214-218.

11 S. D. Melman, M. L. Steinauer, C. Cunningham, L. S. Kubatko, I. N. Mwangi, N. B. Wynn, M. W. Mutuku, D. M. S. Karanja, D. G. Colley, C. L. Black, W. E. Secor, G. M. Mkoji and E. S. Loker, PLoS Neglected Trop. Dis., 2009, 3, e504.

12 P. G. Fallon, R. F. Sturrock, A. C. Niang and M. J. Doenhoff, Am. J. Trop. Med. Hyg., 1995, 53, 61-62.

13 F. F. B. Couto, P. M. Z. Coelho, N. Araújo, J. R. Kusel, N. Katz, L. K. Jannotti-Passos and A. C. A. Mattos, Mem. Inst. Oswaldo Cruz, 2011, 106, 153-157. 
14 P. G. Fallon and M. J. Doenhoff, Am. J. Trop. Med. Hyg., 1994, 51, 83-88.

15 M. M. Ismail, S. A. Taha, A. M. Farghaly and A. S. El-Azony, J. Egypt. Soc. Parasitol., 1994, 24, 685-695.

16 B. J. Neves, R. C. Braga, J. C. B. Bezerra, P. V. L. Cravo and C. H. Andrade, PLoS Neglected Trop. Dis., 2015, 9, e3435.

17 G. E. Torres, R. R. Gainetdinov and M. G. Caron, Nat. Rev. Neurosci., 2003, 4, 13-25.

18 B. J. Hoffman, S. R. Hansson, E. Mezey and M. Palkovits, Front. Neuroendocrinol., 1998, 19, 187-231.

19 T. E. Mansour, Adv. Parasitol., 1985, 23, 1-36.

20 M. S. Rahman, D. F. Mettrick and R. B. Podesta, Exp. Parasitol., 1985, 60, 10-17.

21 J. P. Boyle, J. V. Zaide and T. P. Yoshino, Exp. Parasitol., 2000, 94, 217-226.

22 J. P. Boyle and T. P. Yoshino, J. Parasitol., 2005, 91, 542-550.

23 N. Patocka and P. Ribeiro, Mol. Biochem. Parasitol., 2007, 154, 125-133.

24 a. C. K. Fontana, M. S. Sonders, O. S. Pereira-Junior, M. Knight, J. a. Javitch, V. Rodrigues, S. G. Amara and O. V. Mortensen, Eur. J. Pharmacol., 2009, 616, 48-57.

25 P. Ribeiro and N. Patocka, Parasitol. Int., 2013, 62, 629-638.

26 D. Cioli, L. Pica-Mattoccia, A. Basso and A. Guidi, Mol. Biochem. Parasitol., 2014, 195, 23-29.

27 G. Panic, D. Flores, K. Ingram-Sieber and J. Keiser, Parasites Vectors, 2015, 8, 624.

28 N. Cowan and J. Keiser, Parasites Vectors, 2015, 13, 417.

29 G. Panic, M. Vargas, I. Scandale and J. Keiser, PLoS Neglected Trop. Dis., 2015, 9, e0003962.

30 K. Ingram-Sieber, N. Cowan, G. Panic, M. Vargas, N. R. Mansour, Q. D. Bickle, T. N. C. Wells, T. Spangenberg and J. Keiser, PLoS Neglected Trop. Dis., 2014, 8, e2610.

31 R. A. Paveley and Q. D. Bickle, Parasite Immunol., 2013, 35, 302-313.

32 M.-H. Abdulla, D. S. Ruelas, B. Wolff, J. Snedecor, K.-C. Lim, F. Xu, A. R. Renslo, J. Williams, J. H. McKerrow and C. R. Caffrey, PLoS Neglected Trop. Dis., 2009, 3, e478.

33 N. R. Mansour and Q. D. Bickle, PLoS Neglected Trop. Dis., 2010, 4, e795.

34 N. Patocka and P. Ribeiro, Mol. Biochem. Parasitol., 2013, 187, 32-42.

35 M. Darmon, S. Al Awabdh, M.-B. Emerit and J. Masson, Prog. Mol. Biol. Transl. Sci., 2015, 132, 97-126.
36 T. Schmidt, A. Bergner and T. Schwede, Drug Discovery Today, 2013, 19, 890-897.

37 A. Penmatsa, K. H. Wang and E. Gouaux, Nat. Struct. Mol. Biol., 2015, 22, 506-508.

38 A. Penmatsa, K. H. Wang and E. Gouaux, Nature, 2013, 503, 85-90.

39 M. Marxer, K. Ingram and J. Keiser, Parasites Vectors, 2012, 5, 165.

40 L. Kamentsky, T. R. Jones, A. Fraser, M.-A. Bray, D. J. Logan, K. L. Madden, V. Ljosa, C. Rueden, K. W. Eliceiri and A. E. Carpenter, Bioinformatics, 2011, 27, 1179-1180.

41 D. A. Benson, M. Cavanaugh, K. Clark, I. Karsch-Mizrachi, D. J. Lipman, J. Ostell and E. W. Sayers, Nucleic Acids Res., 2013, 41, D36-D42.

42 M. Biasini, S. Bienert, A. Waterhouse, K. Arnold, G. Studer, T. Schmidt, F. Kiefer, T. G. Cassarino, M. Bertoni, L. Bordoli and T. Schwede, Nucleic Acids Res., 2014, 42, W252-W258.

43 R. A. Laskowski, M. W. MacArthur, D. S. Moss and J. M. Thornton, J. Appl. Crystallogr., 1993, 26, 283-291.

44 C. Colovos and T. O. Yeates, Protein Sci., 1993, 2, 1511-1519.

45 J. C. Shelley, A. Cholleti, L. L. Frye, J. R. Greenwood, M. R. Timlin and M. Uchimaya, J. Comput.-Aided Mol. Des., 2007, 21, 681-691.

46 J. L. Banks, H. S. Beard, Y. Cao, A. E. Cho, W. Damm, R. Farid, A. K. Felts, T. A. Halgren, D. T. Mainz, J. R. Maple, R. Murphy, D. M. Philipp, M. P. Repasky, L. Y. Zhang, B. J. Berne, R. A. Friesner, E. Gallicchio and R. M. Levy, J. Comput. Chem., 2005, 26, 1752-1780.

47 OpenEye Scientific Software Inc. OMEGA v. 2.5.1. Available from: http://www.eyesopen.com, 2013.

48 A. Jakalian, D. B. Jack and C. I. Bayly, J. Comput. Chem., 2002, 23, 1623-1641.

49 OpenEye Scientific Software Inc. QUACPAC v. 1.6.3. Available from: http://www.eyesopen.com, 2013.

50 M. McGann, J. Chem. Inf. Model., 2011, 51, 578-596.

51 OpenEye Scientific Software Inc. OEDocking v. 3.0.1. Available from: http://www.eyesopen.com, 2012.

52 M. R. McGann, H. R. Almond, A. Nicholls, J. A. Grant and F. K. Brown, Biopolymers, 2003, 68, 76-90.

53 M. McGann, J. Comput.-Aided Mol. Des., 2012, 26, 897-906.

54 N. Patocka, N. Sharma, M. Rashid and P. Ribeiro, PLoS Pathog., 2014, 10, e1003878.

55 F. Angelucci, A. E. Miele, G. Boumis, M. Brunori, D. Dimastrogiovanni and A. Bellelli, Curr. Top. Med. Chem., 2011, 11, 2012-2028. 